\title{
OPERATIONS ON BINARY RELATIONS AND THEIR APPLICATIONS ${ }^{1}$
}

\author{
BY T. TAMURA
}

Communicated by Edwin Hewitt, September 27, 1963

1. Introduction. As far as the existence of the smallest congruence of a given type on a semigroup is concerned, Kimura, Yamada and the author discussed special cases of semigroups in $[7 ; 11]$ and identity conditions in [8]; the author argued implication conditions in [9]; Kimura generalized them to algebraic systems [4]. Also, Clifford and Preston interpreted these results of the principle of the maximal homomorphic image in [3]. Although the proof of existence was easily obtained, the problem of constructing the smallest congruence in the general case still remains. In this paper we define types of relations by means of semi-closure operations and discuss the existence of the smallest relation of a given type. In particular, if we provide semi-closure operations with the condition "join conservative," then we can explicitly state the method of construction of the smallest relation of a given type. This paper is a simplification, resystematization, and generalization of the theory in [9].

2. General theory of operations on relations. Let $E$ be a set. A binary relation $\rho$ is a subset of the product set $E \times E$. Let $B$ be a complete lattice composed of binary relations with respect to the usual inclusion relation $\subseteq$. For an arbitrary subset $a=\left\{\rho_{\alpha} ; \alpha \in \Gamma\right\}$ of $B$, the join and meet are denoted by

$$
\bigcup_{\alpha \in \Gamma} \rho_{\alpha} \text { or } J(Q) \text { and } \bigcap_{\alpha \in \Gamma} \rho_{\alpha} \text { or } M(Q)
$$

respectively. $B$ is not required to be the collection of all binary relations. Consider a unary operation $P$, i.e., a mapping of $B$ into itself: $\rho \rightarrow \rho P$. The inclusion relation with respect to the operations is defined as follows:

$$
Q \text { includes } P \text {, i.e., } \quad P \leqq Q \text { means } \rho P \subseteq \rho Q \quad \text { for all } \rho \in Q \text {, }
$$

and hence $P=Q$ iff $\rho P=\rho Q$ for all $\rho \in B$. Accordingly, the join and meet, $\cup_{\xi} P_{\xi}, \cap_{\xi} P_{\xi}$, of a set $\left\{P_{\xi} ; \xi \in \Xi\right\}$ are given as follows:

$$
\rho\left(\bigcup_{\xi} P_{\xi}\right)=U_{\xi} \rho P_{\xi}, \quad \rho\left(\bigcap_{\xi} P_{\xi}\right)=\bigcap_{\xi} \rho P_{\xi} .
$$

\footnotetext{
1 This paper is a rapid report without proof. The proofs will appear in [10].
} 
An operation which maps all relations to the empty relation is called the empty operation. A multiplication of operations $P$ and $Q$ is defined by

$$
P Q: \rho(P Q)=(\rho P) Q \text {. }
$$

Following $[1 ; 5]$ we define the following conditions concerning operations $P$ :

(2.4.1) Isotone: $\rho \subseteq \sigma$ implies $\rho P \subseteq \sigma P$.

(2.4.2) Extensive: $\rho \subseteq \rho P$ for all $\rho \in ß$.

(2.4.3) Idempotent: $P^{2}=P$.

An isotone extensive operation is called a semi-closure operation, and an idempotent semi-closure operation is called a closure operation. The set of all semi-closure operations on $B$ is denoted by $\mathfrak{P}$.

LEMMA 2.1. $\Re$ is a complete lattice with respect to (2.1) or (2.2), and at the same time $\mathfrak{B}$ is a partially ordered semigroup with respect to (2.3) and (2.1), that is, (2.1) is compatible with respect to (2.3).

As usual, if $\rho P=\rho$, then $\rho$ is called $P$-closed.

For any subset of $\mathfrak{P}$, say, $\left\{P_{\xi} ; \xi \in \Xi\right\}$,

$\Lambda P_{\xi}$ means "A relation $\rho$ is $P_{\xi}$-closed, for all $\xi \in \mathbb{Z}$." $\xi$

$\bigvee P_{\xi}$ means "A relation $\rho$ is $P_{\xi}$-closed, for at least one $\xi \in \Xi . "$ $\xi$

If $\rho$ satisfies $\Lambda_{\xi} P_{\xi},|\Xi| \Xi \mid \geqq 1,{ }^{2}$ then $\rho$ is said to be of meet-type $\Lambda_{\xi} P_{\xi}$; if $\rho$ satisfies $\bigvee_{\xi} P_{\xi},|\Xi|>1$, then $\rho$ is said to be of join-type $\bigvee_{\xi} P_{\xi}$. By "type" we mean either a join-type or a meet-type. Clearly $\rho$ is of type $\Lambda_{\xi} P_{\xi}$ iff $\rho$ is $U_{\xi} P_{\xi}$-closed. The smallest relation of type $T$ including $\rho$ is called the relation of type $T$ generated by $\rho$.

THEOREM 2.1 (EXISTENCE THEOREM FOR MEET-TYPES). Let $\rho$ be any relation belonging to $B$, and $\left\{P_{\xi} ; \xi \in \Xi\right\}$ be a fixed system of semi-closure operations on $B$. There exists the relation $\sigma \in ß$ of meet-type $\Lambda_{\xi} P_{\xi}$ generated by $\rho$.

In the same way, semi-closure operations can be defined in any complete lattice. By Lemma 2.1, $\mathfrak{P}$ is a complete lattice, and the mapping $P \rightarrow P Q$, for a fixed $Q$, is a semi-closure operation on $\mathfrak{P}$. If $P Q=P, P$ is said to be $Q$-closed.

For a subset $\left\{P_{\xi} ; \xi \in \mathbb{\Xi}\right\}$ of $\mathfrak{P}$, there is a closure operation $\bar{P}$ such that $\bar{P}$ is the smallest $P_{\xi}$-closed semi-closure operation, for all $\xi \in \Xi$, which includes all $P_{\xi}$. As in the case of relations, $\bar{P}$ is called the closure

${ }^{2} \mid$ 济 is the cardinal of the set $\mathbf{a}$. 
operation generated by the set $\left\{P_{\xi} ; \xi \in \Xi\right\} . \bar{P}$ is given in the following way:

(2.5) $\rho \bar{P}=M\left(\bigotimes_{\rho}\right)$ where $\circledast_{\rho}=\left\{\sigma \in \circledast ; \sigma \supseteq \rho, \sigma P_{\xi}=\sigma\right.$ for all $\left.\xi \in \Xi\right\}$. We denote $\bar{P}$ by $\bar{P}=\llbracket\left\{P_{\xi} ; \xi \in \Xi \rrbracket ;\right.$ if $|\Xi|=1, \bar{P}=\llbracket P \rrbracket$. Accordingly, $\sigma$ in Theorem 2.1 is given by $\sigma=\rho \bar{P}$. Immediately we have

$$
\bar{P}=\llbracket P_{\xi} ; \xi \in \Xi \rrbracket=\llbracket \llbracket U_{\xi} P_{\xi} \rrbracket=\llbracket \llbracket \bigcup_{\xi}^{U} \bar{P}_{\xi} \rrbracket \text { where } \bar{P}_{\xi}=\llbracket P_{\xi} \rrbracket .
$$

THEOREM 2.2 (EXISTENCE THEOREM FOR JOIN-TYPES). The relation $\sigma$ of a given join-type $\mathrm{V}_{\xi \in \Xi} P_{\xi},|\Xi|>1$, generated by $\rho$ exists iff $\rho P^{\prime}$ is of type $\bigvee_{\xi} P_{\xi}$ where $P^{\prime}=\bigcap_{\xi} \bar{P}_{\xi}, \bar{P}_{\xi}=\llbracket P_{\xi} \rrbracket$. If it exists, $\sigma=\rho P^{\prime}$.

To obtain theorems concerning existence of the smallest relation of given type, not restricted to relations including a given relation, we may replace $\rho$ in Theorems $2.1,2.2$ by the smallest relation $\iota$ in $B$.

If we assume "join-conservative" condition on operations, then $\bar{P}$ can be expressed in explicit form.

A subset $Q$ of $Q$ is called an upper half-subsemilattice if for any $\rho$, $\sigma \in Q$, there is $\tau \in Q$ such that $\rho \cup \sigma \subseteq \tau$. An operation $P$ is called joinconservative if, for any upper half-subsemilattice $Q$ of $B$,

$$
[J(Q)] P \subseteq J(Q P) \text { where } a P=\{\rho P ; \rho \in Q\} .
$$

LEMMA 2.2. If each $P_{\xi}$ is join-conservative, $U_{\xi} P_{\xi}$ is join-conservative. The set of all isotone, join-conservative operations on $B$ is a semigroup with respect to the multiplication (2.3).

If $P$ is a join-conservative semi-closure operation, (2.7) is equivalent to

$$
[J(Q)] P=J(Q P) .
$$

ThEOREM 2.3. Let $\left\{P_{\xi} ; \xi \in \Xi\right\}$ be a set of join-conservative semiclosure operations. Then

$$
\llbracket P_{\xi} ; \xi \in \Xi \rrbracket=\bigcup_{\xi, n} P_{\xi_{1}}^{n_{1}} \cdots P_{\xi_{k}}^{n_{k}}, \quad \xi_{i} \in \Xi,
$$

where $n_{i}$ is a positive integer, $P_{\xi_{1}}^{n_{1}} \cdots P_{\xi_{k}}^{n_{k}}$ is a multiplication in the sense of (2.3), and the union runs through all $\xi_{1}, \cdots, \xi_{k} ; n_{1}, \cdots, n_{k}$; $k=1,2, \cdots$.

Theorem 2.4. Let $\left\{P_{i} ; i=1,2, \cdots, n\right\}$ be $a$ set of a finite number of join-conservative closure operations. If $P_{i} P_{j}=P_{i} P_{j} P_{i}$ for all $i, j, i \neq j$, then

$$
\llbracket P_{i} ; i=1,2, \cdots, n \rrbracket=P_{1} P_{2} \cdots P_{n}=P_{i_{1}} \cdots P_{i_{n}},
$$


$i_{1}, \cdots, i_{n}$ being any permutation of $1, \cdots, n$.

3. Implications. By a function $f$ on a set $E$ we mean a mapping which associates with each ordered system $\left(x_{1}, \cdots, x_{n}\right)$ of elements of $E$ a single element $f\left(x_{1}, \cdots, x_{n} ; a_{1}, \cdots, a_{m}\right)$ of $E$ where $a_{1}, \cdots, a_{m}$ are constant elements of $E$. Consider the following conditions on a relation $\rho$.

For fixed functions $f_{\xi}, g_{\xi}, h$ and $k(\xi \in \Xi)$, $f_{\xi}\left(x_{1}, \cdots, x_{n} ; a_{\xi 1}, \cdots, a_{\xi m_{\xi}}\right) \rho g_{\xi}\left(x_{1}, \cdots, x_{n} ; a_{\xi 1}, \cdots, a_{\xi m_{\xi}}\right), \quad \xi \in \Xi$,

$$
\text { implies } h\left(x_{1}, \cdots, x_{n} ; b_{1}, \cdots, b_{l}\right) \rho k\left(x_{1}, \cdots, x_{n} ; b_{1}, \cdots, b_{l}\right) \text {. }
$$

This means that if there are elements $x_{1}, \cdots, x_{n}$ of $E$ satisfying $f_{\xi} \rho g_{\xi}$ for all $\xi \in \Xi$, then $h \rho k$ holds for those $x_{1}, \cdots, x_{n}$. A condition of this form is called an implication. A semi-closure operation $P$ corresponds to an implication as follows:

$$
\rho P=\rho \cup \tau
$$

where $\tau$ is the set of all $\left(h\left(x_{1}, \cdots, x_{n} ; b_{1}, \cdots, b_{l}\right), k\left(x_{1}, \cdots, x_{n}\right.\right.$; $\left.\left.b_{1}, \cdots, b_{l}\right)\right),\left(x_{1}, \cdots, x_{n}\right)$ running through all the systems satisfying $f_{\xi} \rho g_{\xi}$ for all $\xi \in \Xi$, if they exist. We can verify that $P$ is join-conservative, and $\rho$ satisfies the condition (3.1) iff $\rho$ is $P$-closed or of type $P$. The following condition is called the identity or the identity condition.

$$
\begin{array}{r}
h\left(x_{1}, \cdots, x_{n} ; b_{1}, \cdots, b_{l}\right) \rho k\left(x_{1}, \cdots, x_{n} ; b_{1}, \cdots, b_{l}\right) \\
\text { for all } x_{1}, \cdots, x_{n} \in E .
\end{array}
$$

Identities are special cases of implications. Also, a system of implications gives us a join-conservative meet-type. We remark that Theorem 2.4 is available for identities.

EXAMPLES:

(3.4) Reflexive: $x \rho x$.

(3.5) Symmetric: $x \rho y \Rightarrow y \rho x$.

(3.6) Transitive: $x \rho y, y \rho z \Rightarrow x \rho z$.

(3.7) Left compatible in a groupoid: $x \rho y \Rightarrow z x \rho z y$.

(3.8) Let $\rho$ be a congruence. The condition that $G / \rho$ is a commutative semigroup: $(x y) z \rho x(y z), u v \rho v u$, stots.

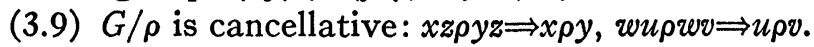

(3.10) $G / \rho$ is weakly reductive [3]: The system of $x a_{\xi} \rho y a_{\xi}, a_{\xi} x \rho a_{\xi} y$, for all $a_{\xi} \in G$, implies $x \rho y$.

The theorems in the preceding section are applied in various ways. For example:

(3.11) Let $\rho$ be a relation on an algebraic system [6]. The smallest 
congruence of a given meet-type including $\rho$ exists. We assume here that an algebraic system has a finite number of binary operations.

(3.12) The smallest equivalence by which an algebraic system is the union of disjoint sub-algebraic-systems exists.

(3.13) In a groupoid ([2] or [3]), the smallest congruence, including a given relation and having the property that the factor groupoid is a partially ordered groupoid, exists.

REMARK. Let $\rho$ be a congruence on a groupoid. The condition " $G / \rho$ contains a right identity" is not a meet-type but a join-type, while the condition "the homomorphic image of a given element $a$ of $G$ is a right identity" is an identity condition. If $G$ is finite, the condition " $G / \rho$ is a group" is a system of identities.

4. Equivalences and congruences. Let $\rho$ be any relation on a set $E$ and $\subseteq$ the set of all relations on $E$. Define $F$ and $S$ as follows:

(4.1) $F: \rho F=\rho \cup \iota$ where $\iota$ is the equality relation.

(4.2) $S: \rho S=\rho \cup \rho(-1)$ where $\rho(-1)=\{(x, y) ;(y, x) \in \rho\}$.

Both $F$ and $S$ are join-conservative closure operations. In the usual way (for example, [3]), we define a binary operation - in $\mathfrak{S}$. If $\rho$, $\sigma \in \mathfrak{S}$,

(4.3) $\rho \cdot \sigma=\{(a, b) ;(a, x) \in \rho,(x, b) \in \sigma$ for some $x \in E\}$ where, if this set is empty, define $\rho \cdot \sigma=\square$, empty relation. Now denote $\rho^{2}=\rho \cdot \rho$.

(4.4) $T_{2}: \rho T_{2}=\rho \cup \rho^{2}$.

$T_{2}$ is a join-conservative semi-closure operation and hence the joinconservative closure operation $T$ generated by $T_{2}$ is given by Theorem 2.3.

(4.5) $T=\llbracket T_{2} \rrbracket=\bigcup_{i=1}^{\infty} T_{2}^{i}$; therefore, $\rho T=\bigcup_{i=1}^{\infty} \rho^{i}$.

The equivalence generated by a relation $\rho$ is given by applying the following join-conservative closure operation to $\rho$.

(4.6) $Q=\llbracket F, S, T \rrbracket, Q$ is called the equivalence operation. Namely, $\rho Q$ is the equivalence generated by $\rho$, and $\sigma$ is an equivalence iff $\sigma=\sigma Q$.

We have the following properties:

(4.7) $F^{2}=F, S^{2}=S, T^{2}=T$;

(4.8) $F S=S F$

(4.9) $F T=T F$;

(4.10) $S T S=T S T=S T$.

Let $\mathfrak{Q}$ be the subsemigroup of $\mathfrak{P}$ (cf. Lemma 2.1) generated by $F, S, T$. It is interesting that $\mathfrak{Q}$ is always finite. For this purpose we can show the free semigroup $\mathfrak{Q}^{*}$ generated by $F, S, T$ with the above relation is finite.

Thus we have: 
ThEOREM 4.1. The free semigroup $\mathfrak{Q}^{*}$ generated by $F, S, T$ with the product relations (4.7) through (4.10) is a partially ordered semigroup composed of nine elements: $F, S, T, S T, T S, F T, F S, F S T, F T S$, with the following partial ordering diagram:

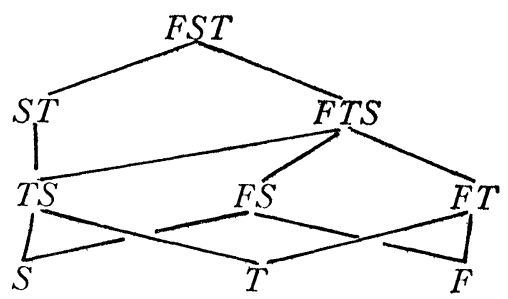

THEOREM 4.2. $Q=F S T$, that is, the equivalence generated by $\rho$ is $\rho(F S T)(c f .[3])$.

REMARK (1). Clearly $Q=F S T=F T S T$, etc. But FST, SFT, STF are the expressions for $Q$ of the smallest length.

Let $\mathfrak{I}^{*}$ be the subsemigroup of $\mathfrak{D}^{*}$ generated by $S$ and $T$. Then

$$
\mathfrak{Q}^{*}=\mathfrak{I}^{*} \cup\{F\} \cup F \mathfrak{T}^{*}
$$

where $\mathfrak{Q}^{*}$ is the union of the three disjoint subsemigroups. Every element of $F \mathfrak{T}^{*}$ is uniquely expressed by the product of $F$ and an element of $\mathfrak{T}^{*}$, and $F$ commutes with every element of $\mathfrak{T}^{*}$. Generally let $A$ and $B$ be arbitrary groupoids, and $A^{1}$ and $B^{1}$ be the groupoids obtained by adjoining the identity 1 . Even if $A$ or $B$ contains the identity already, such a procedure is valid. The direct product $A^{1} \times B^{1}$ from which the identity of $A^{1} \times B^{1}$ is excluded is called the annexed product of $A$ and $B$. Then we can say that $\mathfrak{Q}^{*}$ is the annexed product of $\{F\}$ and $\mathfrak{T}^{*}$.

REMARK (2). If $E$ has a cardinal $\geqq 3$, then $\mathfrak{Q}=\mathfrak{Q}^{*}$.

Let $G$ be a groupoid in the sense of [2] or [3], and let $\rho$ be any relation in $G$. Operations $C_{r}, C_{l}$ and $C$ are defined in the following way.

$$
\begin{gathered}
C_{r} \text { and } C_{l} \\
\rho C_{r}=\rho \cup\{(a x, b x) ; x \in G,(a, b) \in \rho\}, \\
\rho C_{l}=\rho \cup\{(x a, x b) ; x \in G,(a, b) \in \rho\} .
\end{gathered}
$$

$C_{r}$ and $C_{l}$ are join-conservative semi-closure operations. $C$ is defined to be the closure operation generated by $C_{r}$ and $C_{l}$. Then we have

$$
\begin{aligned}
C & =\llbracket C_{r}, C_{l} \rrbracket=\underset{i_{, n}}{\bigcup} C_{i_{1}}^{n_{1}} \cdots C_{i_{k}}^{n_{k}} \quad \text { (where } i_{j} \text { is either } r \text { or } l \text { ) } \\
& =\llbracket C_{R}, C_{L} \rrbracket \quad \text { where } C_{R}=\llbracket C_{r} \rrbracket, \quad C_{L}=\llbracket C_{l} \rrbracket .
\end{aligned}
$$


In particular, if $G$ is a semigroup, then

(4.14) $C_{r}^{2}=C_{r}, C_{l}^{2}=C_{l}, C_{r} C_{l}=C_{l} C_{r}$ and hence $C=C_{r} C_{l}$.

Now we find the form of the operation determining the congruence generated by a relation. Define

(4.15) $N=\llbracket[F, S, C, T \rrbracket, N$ is called the congruence operation.

We have the following product-relations:

(4.16) $F C_{r}=C_{r} F, F C_{l}=C_{l} F$, and hence $F C=C F$;

(4.17) $S C_{r}=C_{r} S, S C_{l}=C_{l} S$, and hence $S C=C S$;

(4.18) $C_{R} T C_{R}=T C_{R} T=C_{R} T, C_{L} T C_{L}=T C_{L} T=C_{L} T$, and hence $C T C=T C T=C T$.

Let $\mathfrak{N}$ be the subsemigroup of $\mathfrak{P}$ generated by $F, S, C, T$. Then $\mathfrak{N}$ is also finite.

TheOREM 4.3. The free semigroup $\mathfrak{N}^{*}$ generated by $S, F, C, T$ with the product relations, (4.7) through (4.10), and the last equalities in (4.16) through (4.18), is the annexed product of $\{F\}$ and $\mathfrak{u}^{*}$ where $\mathfrak{U}^{*}$ is a subsemigroup generated by $S, C, T$ and consists of twelve elements:

$$
S, C, T, S C, S T, T S, C T, T C, S T C, S C T, C T S, T C S .
$$

$\mathfrak{N}^{*}$ is a partially ordered semigroup with twenty-five elements in which FSCT is greatest.

The diagram of $\mathfrak{u}^{*}$ is

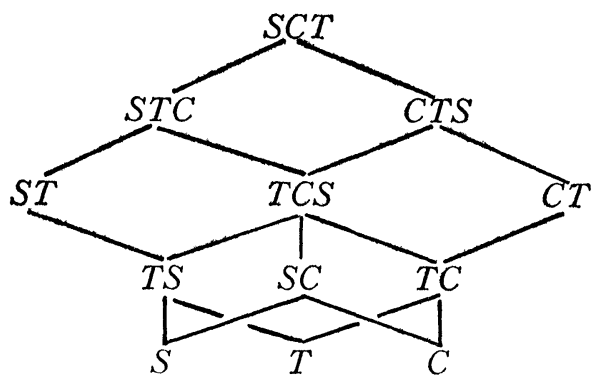

Theorem 4.4. $\rho(F S C T)$ is the congruence generated by $\rho$, that is, $N=F S C T,(c f .[3])$.

REMARK (1). A finite semigroup in which $\mathfrak{N}=\mathfrak{N}^{*}$ exists.

REMARK (2). If $G$ is a semigroup, the free semigroup generated by $F, S, C_{r}, C_{l}, T$, consists of sixty-nine elements.

Finally, we show how to construct a congruence of a given meettype.

THEOREM 4.5. Let $P$ be a join-conservative semi-closure operation. The congruence $\sigma$ of meet-type $P$ generated by $\rho$ is given by 


$$
\sigma=\rho W \quad \text { where } \quad W=\bigcup_{i=1}^{\infty}(P N)^{i}
$$

In particular, if $P$ is a closure operation derived from an identity, then $W=P N$.

REMARK (3). Kimura introduced in [4] a normal property on an algebraic system. A congruence $\rho$ is of meet-type iff $G / \rho$ satisfies a normal property.

We want to add the following fact to the introduction of this paper: Recently Petrich dealt with the greatest semilattice decomposition of a semigroup in Bull. Amer. Math. Soc. 69 (1963), 342-344, independently of [9].

\section{REFERENCES}

1. G. Birkhoff, Lattice theory, rev. ed., Amer. Math. Soc. Colloq. Publ., Vol. 25, Amer. Math. Soc., Providence, R. I., 1948.

2. R. H. Bruck, A survey of binary systems, Springer-Verlag, Berlin, 1958.

3. A. H. Clifford and G. Preston, Algebraic theory of semigroups. Vol. 1, Math. Surveys No. 7, Amer. Math. Soc., Providence, R. I., 1961.

4. N. Kimura, On some existence theorems on multiplicative system. I, Great quotient, Proc. Japan Acad. 34 (1958), 305-309.

5. O. Ore, Some studies on closure relations, Duke Math. J. 10 (1943), 761-785.

6. K. Shoda, Allgemeine Algebra, Osaka Math. J. 1 (1949), 182-225.

7. T. Tamura and N. Kimura, On decompositions of a commutative semigroup, Kodai Math. Sem. Rep. 4 (1954), 109-112.

8. - Existence of greatest decomposition of a semigroup, Kōdai. Math. Sem. Rep. 7 (1955), 83-84.

9. T. Tamura, The theory of construction of finite semigroups. I, Osaka Math. J. 8 (1956), 243-261.

10. - Theory of operations on binary relations (to appear).

11. M. Yamada, On the greatest semilattice decomposition of a semigroup, Ködai Math. Sem. Rep. 7 (1955), 59-62.

University of California, Davis 